\title{
Long term Parenteral Nutrition in children: 10 year experience from a single centre
}

\author{
C. Cole ${ }^{1}$, A. E. Wiskin ${ }^{2}$, M. Cullen ${ }^{2}$ and R. M. Beattie ${ }^{2}$ \\ ${ }^{1}$ Pharmacy department, ${ }^{2}$ Paediatric Medical Unit, Southampton General Hospital, SO16 6YD, UK
}

Southampton provides tertiary services for a population of approximately 650000 children. As part of the complex treatment of children referred to Southampton, a number require Parenteral Nutrition (PN) for greater than 28 days. We describe the trends in prolonged PN usage at our centre over the last 10 years.

Children who received PN for $>28$ days were identified from PN prescriptions managed by pharmacy. Data was collected retrospectively from patient records. Babies who received PN solely on the neonatal unit were excluded as were those who only received PN on the Paediatric Intensive Care Unit.

74 (35 female) children were identified having 81 episodes of PN $>28$ days. New and total number of patients per year, are shown in the table. Median age at start of PN episode was 2.9 years with range of 0 to 18 years. Duration of PN ranged from 29 days to 2108 days (6 years), median duration was 63 days. 30 children had 31 episodes of PN >90 days. 57 children came off PN, ten are currently at home on PN, four children died; two from their oncological diagnosis and two had intractable gut disease. Two children were transferred to adults and one to another centre. In total 18 children received home PN in this 10 year period.19 children received PN due to complications of oncological diagnoses or treatment, 16 due to surgical intra-abdominal crises, 8 for Inflammatory Bowel Disease, 4 for Hirschprung's, 9 for enteropathies and 11 for congenital defects including 7 with gastroschisis and 7 for other reasons.

\begin{tabular}{lcc}
\hline Period & New cases & Total cases \\
\hline Apr 01-Mar 02 & 2 & 3 \\
Apr 02-Mar 03 & 4 & 6 \\
Apr 03-Mar 04 & 7 & 9 \\
Apr 04-Mar 05 & 4 & 5 \\
Apr 05-Mar 06 & 11 & 14 \\
Apr 06-Mar 07 & 5 & 8 \\
Apr 07-Mar 08 & 7 & 10 \\
Apr 08-Mar 09 & 9 & 11 \\
Apr 09-Mar 10 & 13 & 21 \\
Apr 10-Mar 11 & 13 & 21 \\
\hline
\end{tabular}

Prolonged use of PN has increased over the last 10 years with a growing number of children receiving PN at home. Despite increasing numbers, the majority of children come off PN. There is a need for collaborative working with adult PN teams as some children require transfer to adult services. The management of these children is complex and requires a dedicated multidisciplinary team. A sustained rise in incident cases would have implications for those planning regional and national services. 